Gursky, H., Giacconi, R., Gorenstein, P., Waters, J. R., Oda, M., Bradt, H., Garmire, G., and Sreekantan, B. V., $1966 a, A p . J ., 146,310$. $1966 b, A p . J ., 144,1249$.

Humason, M. L. 1938, Ap. J., 88, 228.

Kraft, R. P. 1963, Advances in Astronomy and Astrophysics, ed. Z. Kopal (New York: Academic Press), 2,43 .

1964, Ap. J., 139, 457.

Lenouvel, F. 1957, I.A.U. Symposium 3, Non-Stable Stars, ed. G. Herbig (Cambridge: Cambridge University Press), p. 35.

Manley, O. 1966, Ap.J., 144, 1253.

McLaughlin, D. B. 1953, Ap.J., 117, 279.

Matthews, T. A., and Sandage, A. R. 1963, Ap. J., 138, 30.

Payne-Gaposchkin, C. 1957, The Galactic Novae (Ämsterdam: North-Holland Publishing Co.).

Walker, M. F. 1954, Pub. A.S.P., 66, 71.

Press), p. 46.

. 1962, Photo-electronic Image Devices, ed. McGee, Wilcock, and Mandel (New York: Academic

Press), p. 341.

\title{
THE CHANGE OF INTENSITY, COLOR, LINE STRENGTH, AND LINE POSITION IN THE QSS 3C 446 DURING THE 1966 OUTBURST
}

During the course of routine observations of quasi-stellar radio sources, 3C 446 was found to be abnormally bright on June 24.4 U.T., 1966 (Sandage 1966). A further observation on July 12.4 U.T., 1966, confirmed this result, showing that the object had undergone an outburst of at least $3.2 \mathrm{mag}$. sometime between October 5, 1964, and June 24,1966 . This time interval can be narrowed appreciably because a visual estimate of the $V$ magnitude by Schmidt on September 23 and 25, 1965, gave $V$ not brighter than 18 on those dates. The event is of particular importance as it enables us to make several tests concerning the intensity and position of the emission-line features.

TABLE 1

Photoelectric DATA ON THE OUTBURST IN 3C 446

\begin{tabular}{c|c|c|c}
\hline \hline Date (U.T.) & $V$ & $B-V$ & $U-B$ \\
\hline Oct. $3.2,1964 \ldots \ldots \ldots$ & 1842 & 0.45 & -093 \\
Oct. 5.2, 1964 ...... & 1836 & .43 & -.88 \\
June 24.4, 1966..... & 1514 & 57 & -.51 \\
July 12.4, 1966..... & 1528 & 0.52 & -0.52 \\
\hline
\end{tabular}

Table 1 gives the photoelectric data. The large change in the $U-B$ color is well above the probable error $(\approx \pm 0.03$ of the determination; $B-V$ has also changed but by a smaller, though still significant, amount. We adopt the following mean values in the subsequent discussion. On October 3-5, 1964: $V=18.39, B-V=0.44, U-B=$ -0.90 ; June and July, 1966: $V=15.21, B-V=0.54, U-B=-0.51$. The increase of $0.14 \mathrm{mag}$ in the $V$ magnitude between the June and July observations suggests that the outburst was already then in the decay phase.

To determine the extent of the change in the emission-line intensities, three wellwidened spectrograms were obtained with the Hale 200-inch reflector on July 13.45 U.T., 1966, July 14.44 U.T., 1966, and July 15.40 U.T., 1966, at a dispersion of $190 \AA$ $\mathrm{mm}$. The emission lines of C IV (1550) and C III (1909), originally found by Burbidge (1965) and by Schmidt (1966) to be of strong to moderate intensity before the outburst, 
were not clearly visible, indicating that the contrast between these lines and the continuum had drastically decreased. There was only a faint suggestion to the eye of the C IV (1550) line near $\lambda=3729 \AA$; no trace of the C III (1909) line could be found.

In order to obtain more precise identification and positions of the lines and to measure their intensities, the plates were microphotometered, rectified to intensities using a calibrated wedge, and analyzed by the cross-correlation filtering technique (Westphal 1966) with three different filter widths. Figure 1 shows the result for the plate taken on July 15.40 U.T., 1966. The He comparison spectrum is shown below the filtered QSS spectrum. A feature at $\lambda_{\text {obs }}=3732 \AA$, visible well above the plate noise, is present on all three spectrograms, and we are convinced that it is the C IV (1550) line seen by Burbidge (1965), Schmidt (1966), and Oke (1966). No trace of the C III (1909) line at $\lambda 4585$ was recovered on any of the three plates, and we conclude that it has been submerged in the increased continuum radiation of the outburst.

The equivalent width (E.W.) of the $C$ IV line has been measured on all three filtergrams with the result that E.W. $=9.2,9.3,9.7 \AA$ on the plates obtained at July 13.45 , 14.44, and 15.40 U.T., respectively. These values are quite sensitive to the adopted

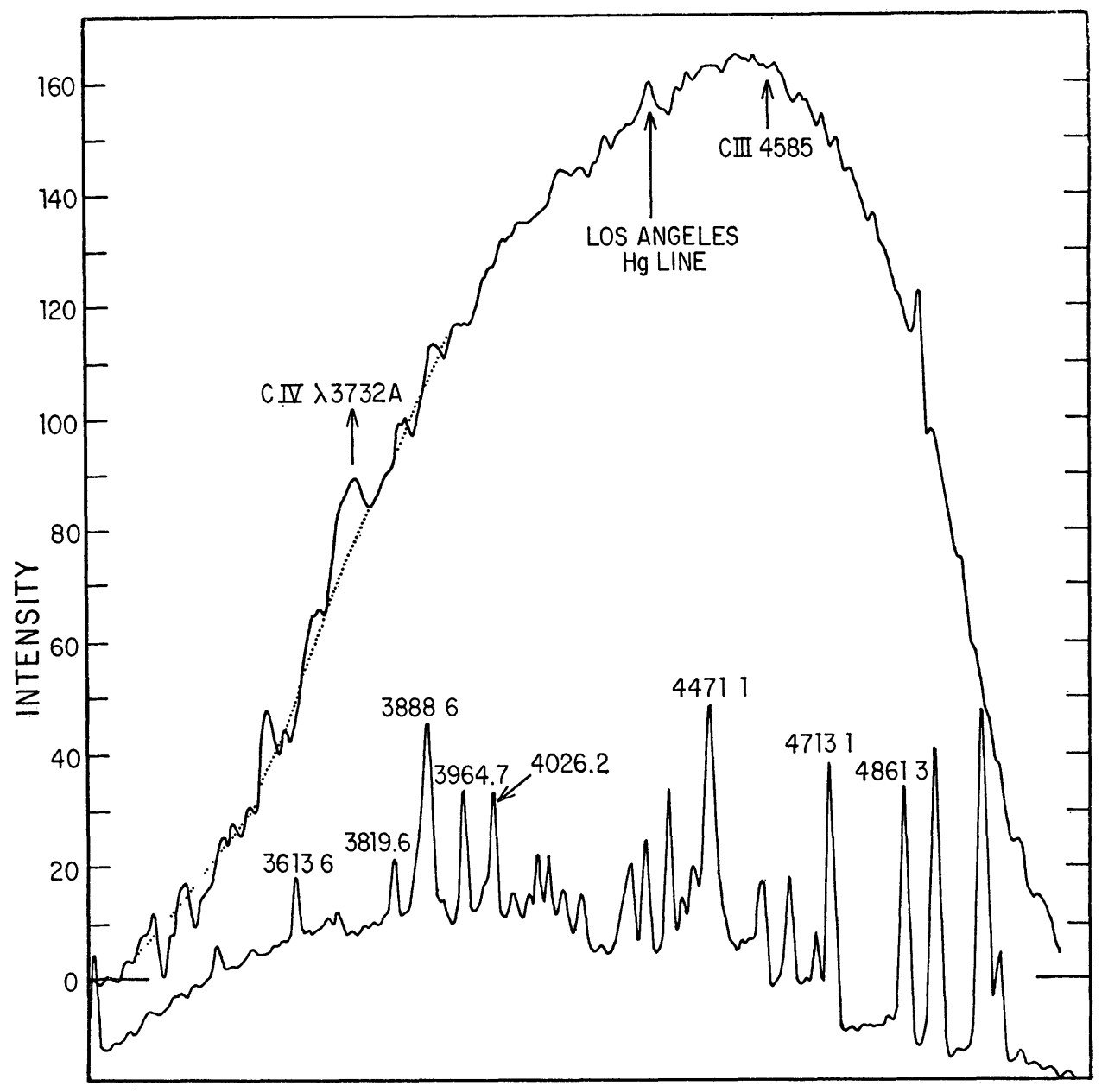

WAVELENGTH

FIG. 1.-Filtered microphotometer record of the spectrogram of 3C 446 taken July 15.40 U.T., 1966. The original digitized output was smoothed with a Gaussian correlation function with a total dispersion width of $13 \AA$. The He comparison lines are shown below the QSS record. The C IV line at $\lambda 3732$ is easily seen. No trace of the $C$ III line expected at $\lambda 4585$ is seen. 
continuum level (see Fig. 1), the resultant uncertainty in equivalent width being perhaps as large as 50 per cent. The equivalent width of $230 \AA$ for C IV (1550) measured in 3C 446 by Oke (1966) before the outburst is much larger than our value. The considerable change in the contrast between line and continuum is beyond doubt and suggests strongly that each is produced in a different region of the object and by unrelated mechanisms.

The digital technique used to filter the microphotometer outputs is ideally suited for the measurement of the wavelength of any desired feature. The He comparison lines are used as a calibration of the wavelength scale in digital units. We sampled the spectrogram at spacings of $1.58 \AA$ which corresponds to every $3 \mu$ on the plate. The computer output permits the determination of the point of maximum correlation on the filtergrams to within one digital unit, or closer if interpolation is desired. This technique was used to determine the wavelength of the maximum correlation of the smoothing filter with the $C$ IV feature, giving $\lambda 3736.4, \lambda 3726.0$, and $\lambda 3733.4$ for the wavelength of the $\mathrm{C}$ IV line on each of the three plates. The mean value is $\lambda 3731.9 \pm 2.8$ (m.e.). This should be compared with Burbidge's (1965) value of $\lambda 3727 \AA$, and Schmidt's (1966) value of $\lambda 3729 \AA$. In view of the line width of at least $50 \AA$, we do not consider the differences to be significant and conclude that there is no evidence for line shifts. The point is of some importance in view of Gold's discussion as summarized by Burbidge and Burbidge (1966).

Finally, there is the question of the colors. We wish to test the hypothesis that the lines have remained essentially constant in absolute intensity, and that the color change is due to the increase in the equivalent width as the continuum intensity changes. We therefore examine the following possibilities: $(a)$ the continuum has increased in intensity by the same amount at all wavelengths and the observed change of color is due solely to the decreased contrast of the lines; $(b)$ the change in color is due to a combination of the effect of the lines and of a change of slope of the continuum energy distribution again keeping the absolute strength of the lines constant. We now show that hypothesis $(a)$ is probably incapable of explaining the observed color changes while $(b)$ gives a self-consistent picture.

Hypothesis (a).-The new observations during outburst give $B-V=+0.54$, $U-B=-0.51$. These colors are consistent with a power-law spectrum $F(\nu) \propto \nu^{-1.8}$ as shown either by Table A4 of Matthews and Sandage (1963, Appendix A) or by direct calculation using Appendix B of the same reference. Since the measured E.W. of C IV (1550) is only about $10 \AA$ at this phase of the outburst, the energy distribution is nearly pure continuum, uncontaminated by the lines We now ask what the E.W. of the C IV (1550) line at $\lambda 3729 \AA$ and $C$ III (1909) at $\lambda 4585 \AA$ would have to have been before the outburst to produce the observed color change of $\Delta(B-V)=0.10 \mathrm{mag}, \Delta(U-B)=$ $0.39 \mathrm{mag}$, if this were the only mechanism operating. The $\lambda 4585$ line affects only $B$, and $\lambda 3729$ affects only $U$. Hence, 0.10 mag must be the contamination factor of the $\lambda 4585$ line in $B$, while $0.49 \mathrm{mag}$ must be the factor in $U$ due to the $\lambda 3729$ line to give $\Delta(U-$ $B)=0.39$ if hypothesis $(a)$ is correct. The E.W. before outburst of the two relevant lines required to cause the observed color change can be calculated from the data given by Strittmatter and Burbidge (1966). They are E.W. $=120 \AA$ for $\lambda 4585$, and E.W. = $380 \AA$ for $\lambda 3729$. The E.W. after outburst in the June-July period should then be these values reduced by the outburst intensity of $3.2 \mathrm{mag}$ (a factor of 19), which gives $\mathrm{E}$.W. (now) $=6 \AA$ for $\lambda 4585$, and E.W. (now) $=20 \AA$ for $\lambda 3729$, again if hypothesis $(a)$ is correct. These four values (before and after outburst) are all high in view of (1) Oke's (1966) measurement of $20 \AA$ for the $\lambda 4585$ line and $230 \AA$ for the $\lambda 3729$ line before outburst, and (2) our measurement of only E.W. (now) $=10 \AA$ for $\lambda 3729$ and our failure to detect the line at $\lambda$ 4585. Furthermore, the required intensity ratio of $\frac{380}{110}$ for the lines before outburst is entirely inconsistent with Oke's (1966) ratio of $\frac{230}{20}$. We feel therefore that hypothesis (a) should be rejected. However, we note that Oke's (1966) photoelectric scans of 3C 446 show several additional features, one of which is identified with 
He II (1640). If all these features are real, the combined equivalent widths under the $U$ and $B$ filters would be more nearly capable of explaining the observed color changes under hypothesis $(a)$. In view of the uncertainty, however, we shall provisionally discount this possibility.

Hypothesis (b).- Here we allow the continuum to change in slope and predict the equivalent width of the $C$ IV (1550) line at $\lambda 3729$ which is required to account for the additional color change. Again we require the total color changes to be $\Delta(B-V)=$ 0.10 and $\Delta(U-B)=0.39$. To simplify, we adopt Oke's pre-outburst value of E.W. $=$ $20 \AA$ for C III (1909). Since the line effectively disappeared in June-July, and hence contributed nothing to the colors at that time, the total change in $B-V$ due to the line change is $0.02 \mathrm{mag}$ (cf. Strittmatter and Burbidge 1966). Hence the remainder of the observed change, namely, $\Delta(B-V)=0.08$ mag must be caused by the change in the slope of the continuum which we henceforth assume to be of a power-law form with index $n\left(F_{\nu} \propto \nu^{-n}\right)$. Referral to Table A4 of Matthews and Sandage (1963) shows that $\Delta(B-V) / \Delta n=0.24$, giving a change in $n$ of $\approx 0.33$ to 1.47 if the color change in $B-V$ is to be explained. The corresponding change in $U-B$ is likewise 0.08 mag.

The change in $U-B$ due to the C IV (1550) line is then calculated as follows. The observed $U-B$ color after outburst was -0.51 minus the effect of the 10 A equivalent width of the $\lambda 3729$ line. This contamination effect is about 0.01 mag giving a continuum color after outburst of $U-B \approx-0.50$ which is to be compared with the observed color of $U-B=-0.90$ before outburst. We have seen that 0.08 mag can be attributed to the change in continuum slope, leaving a difference of $0.32 \mathrm{mag}$ to be explained as the effect on the $U$ magnitude of the $\mathrm{C}$ Iv line. From the work of Strittmatter and Burbidge (1967) we obtain a required equivalent width of C IV (1550) before outburst of $250 \AA$. This is in good agreement with Oke's (1966) measured value of $230 \AA$.

Furthermore, we can predict the equivalent width which the C IV (1550) line should have had in June-July, 1966, if its absolute intensity remained constant. The intensity increase in the $V$ magnitude is a factor $19(3.2 \mathrm{mag})$. The change in slope of the continuum, characterized by a change in exponent from $n=1.8$ to $n=1.47$, means that the intensity increase at $\lambda 3729$ is reduced from 19 to 16.7, giving a predicted equivalent width of $\frac{250}{167}=15 \AA$. Although this is not in perfect agreement with the measured value of about $10 \AA$, we believe the difference is not significant in view of the errors involved both in the estimate of E.W. $=250 \AA$, which is based on the somewhat uncertain observed colors, and in the adopted position of the continuum in Figure 1.

We conclude that the observed change in color is consistent with the hypothesis (i) that the continuum slope became steeper, and the associated colors therefore somewhat redder, during the outburst, and (ii) that the lines remained essentially constant in absolute intensity, changing in equivalent width from about $250 \AA$ to $15 \AA$ for $\mathrm{C}$ IV (1550) at $\lambda 3729$ and from $20 \AA$ to about $1 \AA$ for C III (1909) at $\lambda$ 4585. Detailed spectrum scans will provide a clear test of these conclusions.

The participation of one of us (P. A. S.) was made possible, in part, by a grant from the National Science Foundation.

August 5, 1966

Allan Sandage

J. A. WESTPHAL

P. A. STRITTMATTER

Mount Wilson and Palomar Observatories

CARNEGIE INSTITUTION OF WASHINGTON

CAlifornia Institute of TEChNOLOGY AND

University of California, San Diego

\section{REFERENCES}

Burbidge, E. M. 1965, Ap. J., 142, 1674

Burbidge, E. M., and Burbidge, G. R. 1966, Ap. J., 143, 271. 